University of Nebraska - Lincoln

DigitalCommons@University of Nebraska - Lincoln

12-15-1999

\title{
A patching model for surface tension of spherical droplet and Tolman length. II
}

T.V. Bykov

University of Nebraska-Lincoln

Xiao Cheng Zeng

University of Nebraska-Lincoln, xzeng1@unl.edu

Follow this and additional works at: https://digitalcommons.unl.edu/chemzeng

Part of the Chemistry Commons

Bykov, T.V. and Zeng, Xiao Cheng, "A patching model for surface tension of spherical droplet and Tolman length. II" (1999). Xiao Cheng Zeng Publications. 55.

https://digitalcommons.unl.edu/chemzeng/55

This Article is brought to you for free and open access by the Published Research - Department of Chemistry at DigitalCommons@University of Nebraska - Lincoln. It has been accepted for inclusion in Xiao Cheng Zeng Publications by an authorized administrator of DigitalCommons@University of Nebraska - Lincoln. 


\title{
A patching model for surface tension of spherical droplet and Tolman length. II
}

\author{
T. V. Bykov and X. C. Zeng ${ }^{\text {a) }}$ \\ Department of Chemistry, University of Nebraska-Lincoln, Lincoln, Nebraska 68588
}

(Received 18 August 1999; accepted 23 September 1999)

\begin{abstract}
In the framework of density functional theory (DFT), two patching models for the density profile of spherical liquid droplet are developed. The patching is based on analytical expressions of the asymptote of the density profiles. The first model leads to analytic expressions of the Tolman length and the effective rigidity constant, from which the temperature dependence of the Tolman length and effective rigidity constant can be determined. The second model is developed particularly for small spherical droplets, from which the dependence of chemical potential and the surface tension of the droplet on the radius are obtained. The results are compared with numerical DFT calculations.

(C) 1999 American Institute of Physics. [S0021-9606(99)51847-3]
\end{abstract}

\section{INTRODUCTION}

This article continues the theme of our previous work ${ }^{1}$ (hereafter referred as Paper I), that is, a study of surface tension of liquid droplets and related properties. A motivation of the study is that it is relevant to the gas-liquid nucleation, one of our recent research interests. It is well-known that in the classical theory of homogeneous nucleation ${ }^{2}$ the surface tension of a droplet is taken to be that of planar surface, whereby the dependence of surface tension on the radius of the droplet is not taken into account (the capillarity approximation). It turns out that the resulting rates of the nucleation based on the capillarity approximation can be orders of magnitude different from measured ones in experiments. An improvement over the capillarity approximation is to account for the effect of size dependence of the surface tension. Density functional theory (DFT) of nucleation is such a theory that implicitly incorporates this effect. ${ }^{3,4}$ In fact, DFT is particularly useful to calculate the surface tension in the limit of an infinitely large droplet and to obtain the limiting behavior of related surface properties. ${ }^{5-7}$ However, substantial numerical calculations involved in DFT limit its wide applicability. On the other hand, simple phenomenological theory of nucleation such as the classical one not only offers analytical formulas for evaluating the rate of nucleation but is also easy to apply. Therefore, a wellgrounded analytical formula for the surface tension of a droplet will benefit future development of better phenomenological theory of nucleation.

In Paper I we obtained analytical expressions for the surface tension of planar liquid-vapor interface and the Tolman length within the framework of DFT. We developed a theoretical approach - the patching model for the asymptote of density profile, from which a rational analytical expression for the density profile is obtained. This analytical expression can then be placed into the statistical mechanics formulas for the surface tension and Tolman length. The Tolman length is the coefficient in the first-order curvature correction to the surface tension.

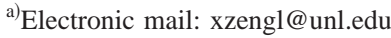

In this work, we will extend the approach in Paper I and study not only the large droplets but also small droplets with size comparable to critical droplets in gas-liquid nucleation at high supersaturation. A different analytical expression for the Tolman length will be present. Moreover, we will present an analytical expression for the second-order curvature correction-the so-called effective rigidity constant. The latter also plays an important part in the dependence of surface tension on the radius of a droplet. Statistical mechanics formulas for both the Tolman length and the effective rigidity constant were originally derived by Blokhuis and Bedeaux. ${ }^{8}$ With the two curvature corrections, the formula for surface tension provides a reasonable description of the size dependence of surface tension of large droplets. We note that these curvature corrections have been calculated numerically ${ }^{9,10}$ and an attempt to find analytical expressions for them has also been made recently by Iwamatsu. ${ }^{11}$

We will introduce an appropriate approximation for the surface tension of relatively small droplets. In this case, the expansion about the (small) curvature is no longer valid. We have considered the size dependence of chemical potential of the droplet and found this dependence for small droplets differs markedly from that obtained from the thermodynamics of nucleation based on the capillarity approximation. This behavior has also been mentioned in a previous DFT study. ${ }^{7}$

In this work, only the Yukawa system is chosen for study, whereas in Paper I both Yukawa and Lennard-Jones systems were studied. For Lennard-Jones systems the involved equations can be so complicated that it is almost impossible to obtain analytical results in many situations. Despite that, we reported in Paper I that although the asymptote of density profile has a different form, expressions for the surface tension of planar liquid-vapor interface and the Tolman length are similar to those of the Yukawa system. We therefore only consider the Yukawa system in this work.

The remainder of the paper is organized in the following way. In Sec. II the patching model based on the functional expansion of density profile about the small curvature is described. Section III gives analytical expressions for the Tolman length and the effective rigidity constant. Section IV 
describes the construction of a patching model for the density profile of relatively small droplets. Section V presents the size dependence of chemical potential as well as the surface tension of a spherical liquid droplet. Finally, the conclusion is given in Sec. VI.

\section{EQUATION FOR ASYMPTOTE OF DENSITY PROFILE OF PLANAR LIQUID-VAPOR INTERFACE AND CURVATURE CORRECTION}

As in Paper I, we first obtain analytical expressions for the asymptotes of density profile. The derived asymptotes will then be used to build a patching model for the whole density profile.

According to DFT ${ }^{12}$ all thermodynamic quantities of the liquid-vapor system can be presented as a functional of the density profile $\rho(\mathbf{r})$. Let $\rho_{b}$ represents the density of bulk phase (liquid or vapor) and $\Delta \rho(\mathbf{r})=\rho(\mathbf{r})-\rho_{b}$ the difference between the density at $\mathbf{r}$ and the bulk density. To obtain the asymptote of density profile near bulk phase, we assume that $\Delta \rho(\mathbf{r}) / \rho_{b}$ is a small quantity. With this assumption, as shown in Paper I, the equation for the asymptote of density profile has the form

$$
\Delta \rho\left(r^{\prime}\right)=\rho_{b} \int d r^{\prime \prime} c^{(2)}\left(\rho_{b}, r\right) \Delta \rho\left(r^{\prime \prime}\right),
$$

where $r=\left|\mathbf{r}^{\prime}-\mathbf{r}^{\prime \prime}\right|$ and $c^{(2)}\left(\rho_{b}, r\right)$ is the second direct correlation function of the bulk phase.

For a large spherical droplet, the density profile $\rho(\mathbf{r})$ can be written as ${ }^{8,10}$

$$
\rho(r)=\rho_{(0)}(z)+\frac{\rho_{(1)}(z)}{R}+\frac{\rho_{(2)}(z)}{R^{2}}+\cdots,
$$

where $z=r-R$ is the distance from the dividing surface, $R$ is the radius of the dividing surface, $\rho_{(0)}(z)$ is the density profile of the planar liquid-vapor interface, and $\rho_{(1)}(z)$, $\rho_{(2)}(z), \ldots$, are the curvature corrections to the density profile. Using expansion (2) and $\Delta \rho(\mathbf{r})=\rho(\mathbf{r})-\rho_{b}$, we can express the density of the system in the form

$$
\begin{aligned}
& \rho_{b}=\rho_{(0) b}+\frac{\rho_{(1) b}}{R}+\frac{\rho_{(2) b}}{R^{2}}+\cdots, \\
& \Delta \rho(r)=\rho_{(0)}(z)+\frac{\Delta \rho_{(1)}(z)}{R}+\frac{\Delta \rho_{(2)}(z)}{R^{2}}+\cdots,
\end{aligned}
$$

where $\rho_{(0) b}$ is the bulk density of the system with planar liquid-vapor interface and $\rho_{(1) b}, \rho_{(2) b}, \ldots$, are curvature corrections to the bulk density. The expansion in (3) is about a small parameter $1 / R$ (if $R$ is dimensionless) or more precisely, about $d / R$ where $d$ is the size of the molecule (in this study, $d$ is taken as the diameter of hard sphere). Clearly, this expansion is valid only for large droplets. We have another expansion as shown in Eqs. (5) and (6) of Paper I. That expansion is about the small parameter $\Delta \rho(r) / \rho_{b}$ and is valid only in the region near bulk phase. Keeping only the zero- and first-order terms in both expansions and using the method proposed by Blokhuis, ${ }^{8}$ one can derive the following two equations from Eq. (1):

$$
\begin{aligned}
\Delta \rho_{(0)}(z)= & 2 \pi \rho_{(0) b} \int_{0}^{\infty} r^{2} d r \int_{-1}^{1} c^{(2)}\left(\rho_{(0) b}, r\right) \\
& \times \Delta \rho_{0}(z+s r) d s
\end{aligned}
$$

and

$$
\begin{aligned}
\Delta \rho_{(1)}(z)= & 2 \pi \rho_{(0) b} \int_{0}^{\infty} r^{2} d r \int_{-1}^{1} c^{(2)}\left(\rho_{(0) b}, r\right)\left[\Delta \rho_{(1)}(z\right. \\
& \left.+s r)+\Delta \rho_{(0)}(z+s r) s r\right] d s .
\end{aligned}
$$

Equations (4) and (5) are the first two equations of a series of integral equations for the asymptote of density profile in the quasiplanar approximation.

To calculate the second direct correlation function appearing in Eqs. (4) and (5), we need a model system and a free-energy functional for the liquid states of that system. In this work we consider only the Yukawa system. Following Paper I, we treat the Yukawa potential function as a hard sphere plus a small attractive potential

$$
w(r)=-\frac{\beta \lambda^{3}}{4 \pi} \frac{\exp (-\lambda r)}{\lambda r},
$$

where $\lambda=1 / d$ and $\beta=-4 \pi \int_{0}^{\infty} d r r^{2} w(r)$ are parameters of the potential. We employed the free-energy functional with local density approximation for the hard spheres and random phase approximation for the attractive part. ${ }^{12,3,4}$ This functional leads to a second direct correlation function given by

$$
\begin{aligned}
c^{(2)}\left(\rho_{(0) b}, r\right)= & \frac{\delta(r)}{\rho_{(0) b}}-\frac{1}{k_{B} T} \frac{\partial \mu_{h}\left(\rho_{(0) b}\right)}{\partial \rho} \delta(r) \\
& -\frac{1}{k_{B} T} w(r),
\end{aligned}
$$

where $\delta(r)$ is the Dirac delta function and $\mu_{h}(\rho)$ is the chemical potential of hard spheres in Carnahan-Starling form. ${ }^{12,3,4}$ Substituting Eq. (7) into Eqs. (4) and (5) yields

$m_{b} \Delta \rho_{(0)}(z)+2 \pi \int_{0}^{\infty} r^{2} d r \int_{-1}^{1} d s w(r) \Delta \rho_{(0)}(z+s r)=0$,

and

$$
\begin{gathered}
m_{b} \Delta \rho_{(1)}(z)+2 \pi \int_{0}^{\infty} r^{2} d r \int_{-1}^{1} d s w(r)\left[\Delta \rho_{(1)}(z+s r)\right. \\
\left.+s r \Delta \rho_{(0)}(z+s r)\right]=0,
\end{gathered}
$$

where $m_{b} \equiv \partial \mu_{h}\left(\rho_{(0) b}\right) / \partial \rho$. The solution of Eq. (8) has been discussed in Paper I. Solving Eq. (9) requires an assumption that the solution can be expressed in the form

$$
\Delta \rho_{(1)}(z)=(a z+b) \Delta \rho_{(0)}(z),
$$

where $a$ and $b$ are two indeterminate parameters. Inserting Eq. (10) into Eq. (9) and using Eq. (8), we have

$(a+1) 2 \pi \int_{0}^{\infty} r^{2} d r \int_{-1}^{1} d s w(r) s r \Delta \rho_{(0)}(z+s r)=0$.

Since the integral in Eq. (11) cannot be zero for the nonuniform system, we have $a=-1$. Thus, Eq. (10) becomes

$$
\Delta \rho_{(1)}(z)=(b-z) \Delta \rho_{(0)}(z)
$$


the parameter $b$ will be determined later using the patching conditions.

To obtain an analytical expression for the first curvature correction of the density profile $\rho_{(1)}(z)$, we used the same patching approach as in Paper I. This approach is based on the continuity of the density profile and its first derivative at the patching point $z_{0}$. For the Yukawa system, this approach gives the density profile of the planar liquid-vapor interface

$$
\begin{aligned}
& \rho_{(0)}(z)=\rho_{(0) 1}+c_{1} \exp \left(-\zeta_{1} \lambda z\right), \quad z>z_{0}, \\
& \rho_{(0)}(z)=\rho_{(0) 2}-c_{2} \exp \left(\zeta_{2} \lambda z\right), \quad z<z_{0}, \\
& c_{1}=\left(\rho_{(0) 2}-\rho_{(0) 1}\right) \frac{\zeta_{2}}{\zeta_{1}+\zeta_{2}} \exp \left(\zeta_{1} \lambda z_{0}\right), \\
& c_{2}=\left(\rho_{(0) 2}-\rho_{(0) 1}\right) \frac{\zeta_{1}}{\zeta_{1}+\zeta_{2}} \exp \left(-\zeta_{2} \lambda z_{0}\right), \\
& z_{0}=d \frac{\zeta_{1}-\zeta_{2}}{\zeta_{1} \zeta_{2}},
\end{aligned}
$$

where $\rho_{(0) 1}$ is the density of vapor at saturation, $\rho_{(0) 2}$ is the density of bulk liquid coexisting with the vapor, and $\zeta_{b}$ $=\left(\partial \mu\left(\rho_{(0) b}\right) / \partial \rho\right)^{1 / 2}\left(m_{b}\right)^{-1 / 2}=\left(\rho_{(0) b}^{2} \chi_{b} m_{b}\right)^{-1 / 2}\left(\right.$ where $\chi_{b}$ is the isothermal compressibility of bulk phase). The patching point $z_{0}$ in Eq. (13) is determined from the equimolar dividing surface condition $\int_{-\infty}^{z_{0}} z(\partial \rho(z) / \partial z) d z+\int_{z_{0}}^{\infty} z(\partial \rho(z) /$ $\partial z) d z=0$. Combining Eqs. (12), (13), and the patching conditions, one can arrive at

$$
\begin{aligned}
& \rho_{(1)}(z)=\rho_{(1) 1}+\left(b_{1}-z\right) c_{1} \exp \left(-\zeta_{1} \lambda z\right), \quad z>z_{0}, \\
& \rho_{(1)}(z)=\rho_{(1) 1}-\left(b_{2}-z\right) c_{2} \exp \left(\zeta_{2} \lambda z\right), \quad z<z_{0}, \\
& b_{1}=\frac{\rho_{(1) 2}-\rho_{(1) 1}}{\rho_{(0) 2}-\rho_{(0) 1}}-\frac{1}{\zeta_{1} \lambda}, \\
& b_{2}=\frac{\rho_{(1) 2}-\rho_{(1) 1}}{\rho_{(0) 2}-\rho_{(0) 1}}+\frac{1}{\zeta_{2} \lambda},
\end{aligned}
$$

where $\rho_{(1) 1}$ and $\rho_{(1) 2}$ are curvature corrections to the density of saturated vapor and liquid, respectively; one can also easily show that ${ }^{8,10} \rho_{(1) b}=\left[2 \sigma_{\infty} /\left(\rho_{(0) 2}-\rho_{(0) 1}\right)\right] \rho_{(0) b}^{2} \chi_{b}$, where $\sigma_{\infty}$ is the surface tension of the planar liquid-vapor interface. Having derived Eq. (14), the patching model for the first curvature correction to the density profile of the planar liquid-vapor interface is fully established. This correction has been evaluated numerically in Ref. 10. Analytical expression for this correction was also derived by Blokhuis, ${ }^{13}$ for which he used a symmetric density profile with a freeenergy functional under the gradient expansion approximation. However, these two approximations result in a zero value of the Tolman length.

\section{TOLMAN LENGTH AND EFFECTIVE RIGIDITY CONSTANT}

In Paper I we obtained analytical expressions for the surface tension of the planar liquid-vapor interface and the Tolman length by using the statistical mechanics formulas of Blokhuis and Bedeaux ${ }^{8}$ and the patching model for density profile of planar liquid-vapor interface. The Blokhuis and
Bedeaux's formulas (with the random phase approximation) for the surface tension and the Tolman length are

$$
\begin{aligned}
\sigma_{\infty}= & \frac{\pi}{2} \int_{-\infty}^{\infty} d z \int_{0}^{\infty} d r r^{3} \frac{\partial w(r)}{\partial r} \int_{-1}^{1} d s\left(1-3 s^{2}\right) \rho_{(0)}(z) \\
& \times \rho_{(0)}(z+s r),
\end{aligned}
$$

and

$$
\begin{aligned}
\delta_{\infty}= & -\frac{\pi}{4 \sigma_{\infty}} \int_{-\infty}^{\infty} d z \int_{0}^{\infty} d r r^{3} \frac{\partial w(r)}{\partial r} \int_{-1}^{1} d s\left(1-3 s^{2}\right)(2 z \\
& +s r) \rho_{(0)}(z) \rho_{(0)}(z+s r) .
\end{aligned}
$$

In this work, with the first curvature correction of the density profile $\rho_{(1)}(z)$ from the patching model, we are able to obtain analytic formulas for the Tolman length $\delta_{\infty}$ and effective rigidity constant $\kappa(\kappa$ is related to the usual rigidity constants $k$ and $\bar{k}$ as $\left.\kappa=(2 k+\bar{k}) / \sigma_{\infty}\right)$ by using two other formulas of Blokhuis and Bedeaux. Specifically, with the random phase approximation these two formulas are given by ${ }^{8,10}$

$$
\begin{aligned}
\delta_{\infty}= & -\frac{\pi}{4 \sigma_{\infty}} \int_{-\infty}^{\infty} d z \int_{0}^{\infty} d r r^{3} \frac{\partial w(r)}{\partial r} \int_{-1}^{1} d s\left(1-3 s^{2}\right) \\
& \times\left[\rho_{(0)}(z) \rho_{(1)}(z+s r)+\rho_{(1)}(z) \rho_{(0)}(z+s r)\right]
\end{aligned}
$$

and

$$
\begin{aligned}
\kappa= & \frac{\pi}{4 \sigma_{\infty}} \int_{-\infty}^{\infty} d z \int_{0}^{\infty} d r r^{3} \frac{\partial w(r)}{\partial r} \int_{-1}^{1} d s\left[\left(1-3 s^{2}\right)(2 z\right. \\
& +s r)\left(\rho_{(0)}(z) \rho_{(1)}(z+s r)+\rho_{(1)}(z) \rho_{0}(z+s r)\right) \\
& \left.-\frac{r^{2} s^{2}}{3}\left(3-5 s^{2}\right) \rho_{(0)}(z) \rho_{(0)}(z+s r)\right]
\end{aligned}
$$

Note that both Eqs. (16) and (18) are only valid when the equimolar dividing surface is located at $z=0$. At this dividing surface, the surface tension of large droplets can be written as

$$
\sigma_{e}\left(R_{e}\right)=\sigma_{\infty}\left(1-2 \delta_{\infty} / R_{e}+\kappa / R_{e}^{2}+\cdots\right),
$$

where $R_{e}$ is the radius of equimolar dividing surface and $\sigma_{e}$ is the surface tension at $R_{e}$. In contrast, the Tolman's formula for the surface tension is valid at any dividing surface and thus the limiting value of the Tolman length $\delta_{\infty}$ is independent of the choice of dividing surface. However, when both the first and the second terms in the expansion of the surface tension about $1 / R$ are involved, the second curvature coefficient will depend on the choice of the dividing surface. Therefore, Eq. (19) is only applicable for the surface tension at equimolar dividing surface.

Another often used dividing surface is the surface of tension. At this surface the surface tension $\sigma_{s}$, the radius of tension $R_{s}$, and the pressure of the uniform liquid and vapor, $p_{2}$ and $p_{1}$, can be related via the simple Laplace equation

$$
p_{2}-p_{1}=2 \sigma_{s}\left(R_{s}\right) / R_{s} \text {. }
$$

In view of the relation between $R_{s}$ and $R_{e}$, the $\sigma_{s}$ can be expressed by

$$
\sigma_{s}\left(R_{s}\right)=\sigma_{\infty}\left(1-2 \delta_{\infty} / R_{s}+\left(\kappa+\delta_{\infty}^{2}\right) / R_{s}^{2}+\cdots\right) .
$$


For the Yukawa system, the integrals in Eqs. (15)-(18) can be carried out analytically when the density profile and its curvature correction are given [Eqs. (13) and (14)]. As we have shown in Paper I the surface tension [using Eq. (15)] is given by

$$
\sigma_{\infty}=\left(\rho_{(0) 2}-\rho_{(0) 1}\right)^{2} \frac{\zeta_{1} \zeta_{2}}{\zeta_{1}+\zeta_{2}} \frac{\beta d}{2} l\left(\zeta_{1}, \zeta_{2}\right)
$$

where $l\left(\zeta_{1}, \zeta_{2}\right)=\left[\zeta_{1}^{2} \zeta_{2}+\zeta_{2}^{2} \zeta_{1}+4 \zeta_{1} \zeta_{2}+2 \zeta_{1}^{2}+2 \zeta_{2}^{2}+4 \zeta_{1}+4\right.$ $\left.\zeta_{2}+2\right] / 2\left(\zeta_{1}+1\right)^{2}\left(\zeta_{2}+1\right)^{2}$, and the Tolman length [using Eq. (16)] is given by

$$
\delta_{\infty}=d \frac{\left(\zeta_{2}-\zeta_{1}\right)}{2 \zeta_{1} \zeta_{2}} \widetilde{l}\left(\zeta_{1}, \zeta_{2}\right)
$$

where $\tilde{l}\left(\zeta_{1}, \zeta_{2}\right)=\left[\left(\zeta_{1}+\zeta_{2}\right)\left(\zeta_{1}^{2}+\zeta_{2}^{2}+\zeta_{1} \zeta_{2}+3 \zeta_{1}+3 \zeta_{2}+3\right)\right.$ $\left.-\zeta_{1} \zeta_{2}+1\right] /\left(\zeta_{1}+1\right)^{3}\left(\zeta_{2}+1\right)^{3} l\left(\zeta_{1}, \zeta_{2}\right)$.

Alternatively, with the first curvature correction to the density profile at hand we can also obtain a different expression of Tolman length from Eqs. (17) and (22); that is,

$$
\delta_{\infty}=-\frac{\rho_{(1) 2}-\rho_{(1) 1}}{\rho_{(0) 2}-\rho_{(0) 1}}-d \frac{\left(\zeta_{2}-\zeta_{1}\right)}{2 \zeta_{1} \zeta_{2}} \widetilde{l}\left(\zeta_{1}, \zeta_{2}\right) .
$$

As mentioned in Paper I, both functions $l\left(\zeta_{1}, \zeta_{2}\right)$ and $\tilde{l}\left(\zeta_{1}, \zeta_{2}\right)$ change slowly with the temperature and both approach a limiting value 1 as $T \rightarrow T_{c}$ (where $T_{c}$ is the critical temperature). Therefore, the temperature dependence of the Tolman length is mainly controlled by terms other than $\widetilde{l}$ in Eq. (24). It is interesting to point out that the simple meanfield formula for the Tolman length ${ }^{13}$ [analogous to Eq. (17)]

$$
\delta_{\infty}=-\frac{2 m}{\sigma_{\infty}} \int_{-\infty}^{\infty} d z \frac{\partial \rho_{(0)}(z)}{\partial z} \frac{\partial \rho_{(1)}(z)}{\partial z},
$$

where $m=\pi / 15 \int_{0}^{\infty} d r r^{5}[\partial w(r) / \partial r]$, combining with $\sigma_{\infty}$ $=\left(\rho_{2}-\rho_{1}\right)^{2} \zeta_{1} \zeta_{2} /\left(\zeta_{1}+\zeta_{2}\right)(\beta d / 2)$ gives

$$
\delta_{\infty}=-\frac{\rho_{(1) 2}-\rho_{(1) 1}}{\rho_{(0) 2}-\rho_{(0) 1}}-d \frac{\left(\zeta_{2}-\zeta_{1}\right)}{2 \zeta_{1} \zeta_{2}},
$$

which would be the same as Eq. $(24)$ if $\widetilde{l}\left(\zeta_{1}, \zeta_{2}\right)=1$. Moreover, recalling $\rho_{(1) 2}-\rho_{(1) 1}=2 \sigma_{\infty} /\left(\rho_{(02) 2}-\rho_{(0) 1}\right)\left(\rho_{(0) 2}^{2} \chi_{2}\right.$ $\left.-\rho_{(0) 1}^{2} \chi_{1}\right)$ and using Eq. (22), we can rewrite both Eqs. (24) and (26) as

$$
\begin{aligned}
\delta_{\infty}= & -\frac{\sigma_{\infty}}{\left(\rho_{(0) 2}-\rho_{(0) 1}\right)^{2}}\left[\left(2-\frac{l\left(\zeta_{1}, \zeta_{2}\right)}{\widetilde{l}\left(\zeta_{1}, \zeta_{2}\right)} k\left(\rho_{(0) 2}\right)\right) \rho_{(0) 2}^{2} \chi_{2}\right. \\
& \left.-\left(2-\frac{l\left(\zeta_{1}, \zeta_{2}\right)}{\widetilde{l}\left(\zeta_{1}, \zeta_{2}\right)} k\left(\rho_{(0) 1}\right)\right) \rho_{(0) 1}^{2} \chi_{1}\right],
\end{aligned}
$$

where $k\left(\rho_{b}\right)=m_{b} / \beta$, and

$$
\begin{aligned}
\delta_{\infty}= & -\frac{\sigma_{\infty}}{\left(\rho_{(0) 2}-\rho_{(0) 1}\right)^{2}}\left[\left(2-k\left(\rho_{(0) 2}\right)\right) \rho_{(0) 2}^{2} \chi_{2}-(2\right. \\
& \left.\left.-k\left(\rho_{(0) 1}\right)\right) \rho_{(0) 1}^{2} \chi_{1}\right] .
\end{aligned}
$$

We discussed in Paper I that Eqs. (27) and (28) can be viewed as general expressions for the Tolman length. Iwamatsu ${ }^{11}$ arrived at a similar formula but used a simple square-gradient approximation for the free-energy functional

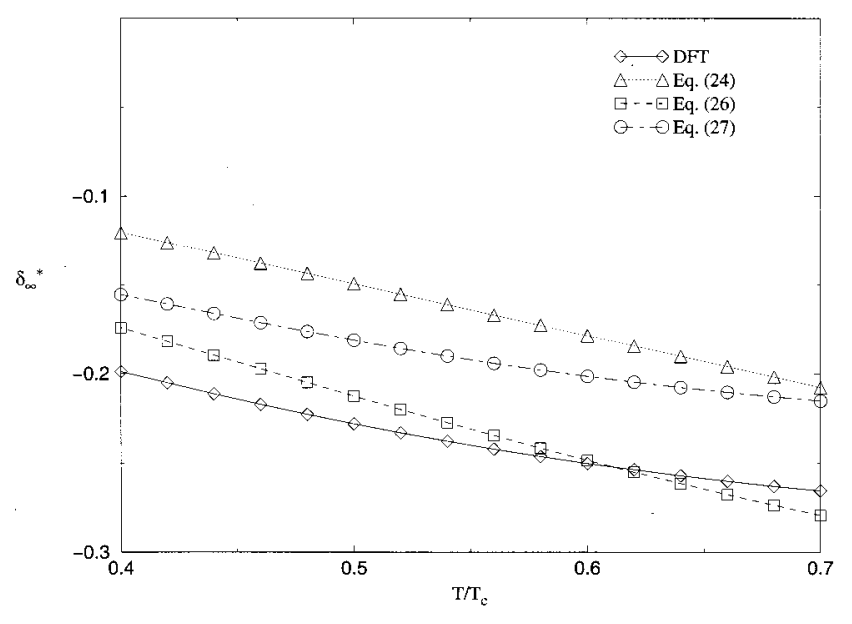

FIG. 1. The scaled Tolman length $\delta_{\infty}^{*}=\delta_{\infty} / d$ versus the scaled temperature $T / T_{c}$.

which may lead to unphysical values of $\zeta_{b}$ at low temperatures (i.e., $\zeta_{b}>1$ ). Compared to Eq. (28), Iwamatsu's formula does not include the term $\left(2-k\left(\rho_{(0) b}\right)\right)$. Note also that Iwamatsu's formula can be derived from another mean-field formula: $\delta_{\infty}=-2 m / \sigma_{\infty} \int_{-\infty}^{\infty} d z z(\partial \rho(z) / \partial z)^{2}$ plus the squaregradient free-energy functional. If the patching model was used, Iwamatsu's formula would be similar to another formula by Blokhuis for the Tolman length.

In Fig. 1 the temperature dependence of the Tolman length $\delta_{\infty}$ is shown. The four curves correspond, respectively, to the numerical DFT calculations with Eq. (17), ${ }^{10} \mathrm{Eq}$. (24), Eq. (26), and Eq. (27) with numerical DFT values of $\sigma_{\infty}$. It can be seen that all curves lie close to each other. In particular, the shape and the bend of the curve from Eq. (27) is very similar to the curve from DFT calculations. All four curves show that the Tolman length is a small negative quantity with its absolute value weakly increasing with temperature. The same result was obtained in Paper I using the first formula of Blokhuis (16).

One may notice that results of Tolman length shown in Fig. 1 are in excellent agreement with those shown in Fig. 3 of Paper I. This is because these results originate from two equivalent formulas proved by Blokhuis and Bedeaux. This equivalence is somewhat lost [between Eqs. (23) and (24)] due to the patching approximation made for the density profile. However, the less than 5\% difference in numerical values indicates that the proposed patching approximations are quite accurate.

To obtain the effective rigidity constant, we substituted the density profile and its correction (13) and (14) into Eq. (18)

$$
\begin{aligned}
\kappa= & \frac{\rho_{(1) 2}-\rho_{(1) 1}}{\rho_{(0) 2}-\rho_{(0) 1}} \frac{d\left(\zeta_{1}-\zeta_{2}\right)}{\zeta_{1} \zeta_{2}} \widetilde{l}\left(\zeta_{1}, \zeta_{2}\right) \\
& -\frac{d^{2}\left(\zeta_{1}^{2}+\zeta_{2}^{2}-\zeta_{1} \zeta_{2}\right)}{\zeta_{1}^{2} \zeta_{2}^{2}} g\left(\zeta_{1}, \zeta_{2}\right)-d^{2} \widetilde{g}\left(\zeta_{1}, \zeta_{2}\right),
\end{aligned}
$$

where

$$
g\left(\zeta_{1}, \zeta_{2}\right)=\left[l\left(\zeta_{1}, \zeta_{2}\right)\left(\zeta_{1}+1\right)^{4}\left(\zeta_{2}+1\right)^{4}\right]^{-1}
$$




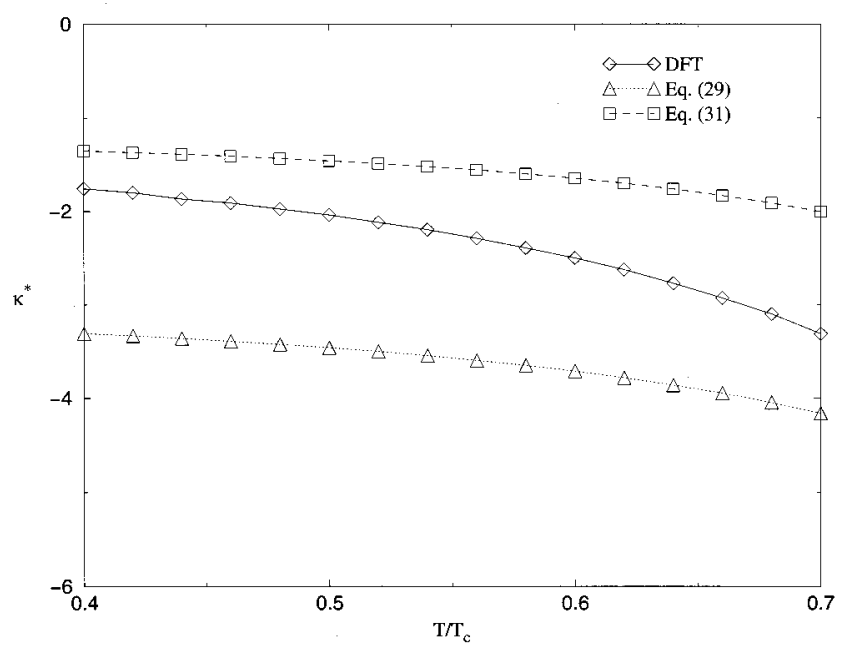

FIG. 2. The scaled effective rigidity constant $\kappa^{*}=\kappa / d^{2}$ versus the scaled temperature $T / T_{c}$.

and

$$
\begin{aligned}
\tilde{g}\left(\zeta_{1}, \zeta_{2}\right)= & {\left[2\left(\zeta_{1}^{6}+\zeta_{2}^{6}\right)+8\left(\zeta_{1}^{5}+\zeta_{2}^{5}\right)+12\left(\zeta_{1}^{4}+\zeta_{2}^{4}\right)\right.} \\
& +8\left(\zeta_{1}^{3}+\zeta_{2}^{3}\right)+\zeta_{1}^{5} \zeta_{2}^{5}\left(\zeta_{1}+\zeta_{2}\right)+10 \zeta_{1} \zeta_{2} \\
& \times\left(\zeta_{1}^{4}+\zeta_{2}^{4}\right)+8 \zeta_{1}^{2} \zeta_{2}^{2}\left(\zeta_{1}^{4}+\zeta_{2}^{4}\right)+2 \zeta_{1} \zeta_{2} \\
& \times\left(\zeta_{1}^{5}+\zeta_{2}^{5}\right)+18 \zeta_{1} \zeta_{2}\left(\zeta_{1}^{3}+\zeta_{2}^{3}\right)+12 \zeta_{1} \zeta_{2} \\
& \times\left(\zeta_{1}^{2}+\zeta_{2}^{2}\right)+86 \zeta_{1}^{3} \zeta_{2}^{3}+7 \zeta_{1}^{3} \zeta_{2}^{3}\left(\zeta_{1}^{3}+\zeta_{2}^{3}\right) \\
& +4 \zeta_{1}^{4} \zeta_{2}^{4}\left(\zeta_{1}^{2}+\zeta_{2}^{2}\right)+12 \zeta_{1}^{2} \zeta_{2}^{2}+34 \zeta_{1}^{2} \zeta_{2}^{2}\left(\zeta_{1}^{3}+\zeta_{2}^{3}\right) \\
& +58 \zeta_{1}^{2} \zeta_{2}^{2}\left(\zeta_{1}^{2}+\zeta_{2}^{2}\right)+23 \zeta_{1}^{4} \zeta_{2}^{4}\left(\zeta_{1}+\zeta_{2}\right) \\
& +36 \zeta_{1}^{3} \zeta_{2}^{3}\left(\zeta_{1}^{2}+\zeta_{2}^{2}\right)+77 \zeta_{1}^{3} \zeta_{2}^{3}\left(\zeta_{1}+\zeta_{2}\right) \\
& \left.+56 \zeta_{1}^{4} \zeta_{2}^{4}+8 \zeta_{1}^{5} \zeta_{2}^{5}+46 \zeta_{1}^{2} \zeta_{2}^{2}\left(\zeta_{1}+\zeta_{2}\right)\right] / \\
& 2 l\left(\zeta_{1}, \zeta_{2}\right) \zeta_{1}^{2} \zeta_{2}^{2}\left(\zeta_{1}+1\right)^{4}\left(\zeta_{2}+1\right)^{4} .
\end{aligned}
$$

The function $g\left(\zeta_{1}, \zeta_{2}\right)$ also approaches to the limiting value 1 as $T \rightarrow T_{c}$ but $\widetilde{g}\left(\zeta_{1}, \zeta_{2}\right)$ approaches to zero as $T \rightarrow T_{c}$. A similar expression of $\kappa$ can be derived from the mean-field formula: ${ }^{13}$

$$
\kappa=\frac{4 m}{\sigma_{\infty}} \int_{-\infty}^{\infty} d z z \frac{\partial \rho_{(0)}(z)}{\partial z} \frac{\partial \rho_{(1)}(z)}{\partial z},
$$

which leads to

$$
\kappa=\frac{d^{2}}{\zeta_{1} \zeta_{2}}\left[\frac{\rho_{(1) 2}-\rho_{(1) 1}}{\rho_{(0) 2}-\rho_{(0) 1}}\left(\zeta_{1}-\zeta_{2}\right)-\frac{\zeta_{1}^{2}+\zeta_{2}^{2}-\zeta_{1} \zeta_{2}}{\zeta_{1} \zeta_{2}}\right] .
$$

If the free-energy functional with square-gradient approximation is used, Eq. (31) is reduced to $\kappa=-d^{2} / \zeta_{1} \zeta_{2}$, the one obtained by Iwamatsu. ${ }^{11}$ In Fig. 2, three curves for the dependence of the effective rigidity constant on the temperature are shown. They are, respectively, from numerical DFT calculation using Eq. (18) ${ }^{10}$ and from formulas (29) and (31). One can see from Fig. 2 that the agreement is reasonable, but not as satisfactory as in the case of the Tolman length.

Using the analytical expressions for the Tolman length and effective rigidity constant plus Eq. (19), one can obtain

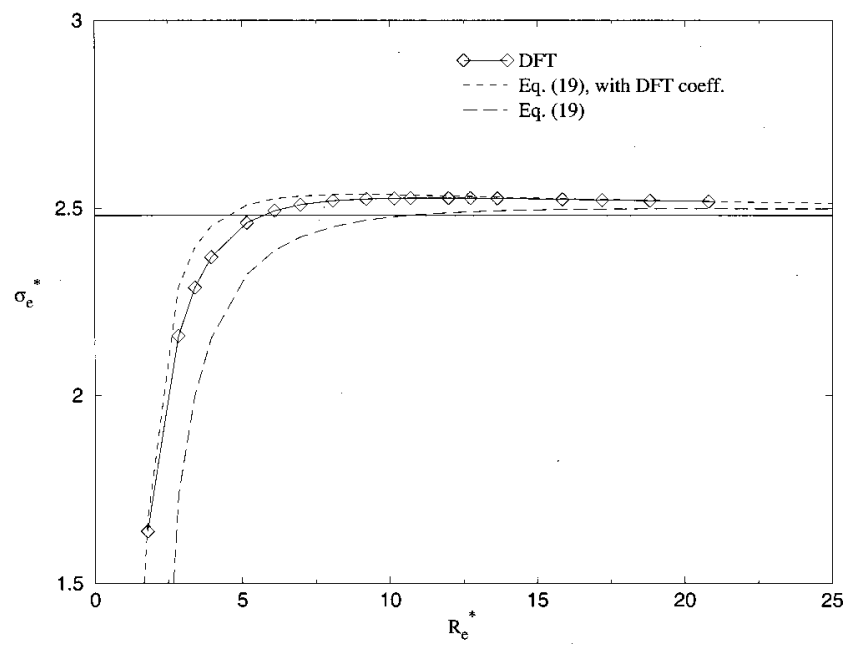

FIG. 3. The scaled surface tension at the equimolar dividing surface $\sigma_{e}^{*}$ $=(\pi / 6)\left(d^{2} / k_{B} T\right) \sigma_{e}$ versus the reduced radius of the equimolar dividing surface $R_{e}^{*}=R_{e} / d$ at $T / T_{c}=0.4$. Horizontal line shows the surface tension of planar liquid-vapor interface.

the dependence of surface tension of a large droplet on the radius. The results are shown in Fig. 3. Two other curves are shown in Fig. 3: one is from numerical DFT calculation of coefficients $\delta_{\infty}$ and $\kappa^{10}$ and the other is also from numerical DFT calculation for the spherical droplet but using a different statistical mechanics formula for the surface tension at equimolar dividing surface, ${ }^{7}$ i.e.,

$$
\sigma(R)=\frac{\Omega+p_{1} V}{4 \pi R^{2}}+\left(p_{2}-p_{1}\right) \frac{R}{3}
$$

where $\Omega$ is the grand canonical potential, $V$ is the volume of the system, $p_{1}$ is the pressure of the uniform vapor, and $p_{2}$ is the pressure of the uniform liquid. One can seen from Fig. 3 that surface tensions evaluated from Eq. (19) are qualitatively in good agreement with DFT results, especially for large droplets. The agreement is even better if the curvature coefficients are calculated numerically. The approximation for the surface tension with two curvature terms [Eq. (19)] was also proposed by Baidakov and Boltachev, ${ }^{9}$ but their numerical calculations were based on square-gradient freeenergy functional.

To close this section, we remark that clearly, the expansion about the small curvature cannot work in the case of small droplets. In the next sections we will introduce a proper approximation for the surface tension of small droplets.

\section{PATCHING MODEL FOR DENSITY PROFILE OF A SMALL SPHERICAL DROPLET}

To develop a patching model for the density profile of a small spherical droplet, we still start from Eq. (1) to obtain an analytical expression for the asymptote of the density profile. For the Yukawa system, once again Eq. (7) is used for the second direct correlation function of uniform system. In this case, Eq. (1) takes the form

$$
m_{b} \Delta \rho(\mathbf{r})+\int d r^{\prime} w\left(\left|\mathbf{r}-\mathbf{r}^{\prime}\right|\right) \Delta \rho\left(\mathbf{r}^{\prime}\right)=0 .
$$


For spherical droplets, Eq. (33) can be written as

$m_{b} \Delta \rho(r)+\frac{2 \pi}{r} \int_{0}^{\infty} d r^{\prime} r^{\prime} \Delta \rho\left(r^{\prime}\right) \int_{\left|r-r^{\prime}\right|}^{\left|r+r^{\prime}\right|} d y y w(y)=0$.

Let $f(r)=\Delta \rho(r) r$. Equation (34) can be further written as

$$
\begin{aligned}
& m_{b} f(r)+2 \pi \int_{0}^{r} d r^{\prime} f\left(r^{\prime}\right) \int_{r-r^{\prime}}^{r+r^{\prime}} d y y w(y) \\
& \quad+2 \pi \int_{r}^{\infty} d r^{\prime} f\left(r^{\prime}\right) \int_{r^{\prime}-r}^{r+r^{\prime}} d y y w(y)=0 .
\end{aligned}
$$

Using Eq. (6) for $w(y)$ and taking the integration by parts repeatedly for an infinite number of times in Eq. (35), one arrives at

$$
\begin{aligned}
& m_{b} f(r)-\beta\left[\sum_{k=0}^{\infty} \lambda^{-2 k} f^{(2 k)}(r)-\sum_{k=0}^{\infty} \lambda^{-2 k} f^{(2 k)}(0)\right. \\
& \quad \times \exp (-\lambda r)]=0,
\end{aligned}
$$

where $f^{(2 k)}(r)$ denotes $2 k$ th derivative of $f(r)$.

For small droplets, we will focus on two regions on the density profile: the region near the center of the droplet and the vapor region at large $r$. Based on our previous results and the work of Iwamatsu, ${ }^{11}$ it seems reasonable to assume that $f(r)$ can be written as a linear combination of exponentials. With this assumption, $f(r)$ at the region near the center of droplet can be chosen as

$$
f_{2}(r)=-c_{2} \sinh \left(\zeta_{2} \lambda r\right),
$$

where $c_{2}$ is a parameter to be determined by using the patching conditions. This choice of $f_{2}(r)$ greatly simplifies Eq. (36) because the second term in the bracket becomes zero. For the vapor region at large $r, f(r)$ takes the form

$$
f_{1}(r)=c_{1} \exp \left(-\zeta_{1} \lambda r\right)
$$

where $c_{1}$ is a parameter to be determined from the patching conditions. For $f_{1}(r)$ at large $r$ the second term in the bracket in Eq. (36) can be neglected since it is much less than the first term. Consequently, in both regions Eq. (36) is reduced to

$$
m_{b}-\beta \sum_{k=0}^{\infty} \zeta_{b}^{2 k}=0
$$

Note that the infinite series summation in Eq. (39) can be easily carried out if $\zeta_{b}<1$, and in that case

$$
m_{b}-\beta\left(1-\zeta_{b}^{2}\right)^{-1}=0,
$$

or

$$
\zeta_{b}=\left(\frac{\partial \mu\left(\rho_{b}\right)}{\partial \rho}\right)^{1 / 2} m_{b}^{-1 / 2}=\left(\rho_{b}^{2} \chi_{b} m_{b}\right)^{-1 / 2} .
$$

Indeed, only values of $\zeta_{b}$ less than 1 are physically meaningful. Otherwise, the density profile would change rapidly for $r$ much greater than $d$.

Given Eqs. (37) and (38), the density profile of the spherical droplet can be written as

$$
\begin{array}{ll}
\rho(r)=\rho_{2}-c_{2} \frac{\sinh \left(\zeta_{2} \lambda r\right)}{r}, & r<r_{0}, \\
\rho(r)=\rho_{1}+c_{1} \frac{\exp \left(-\zeta_{1} \lambda r\right)}{r}, & r>r_{0},
\end{array}
$$

where $r_{0}$ is the patching point. We note that with the squaregradient approximation for the free-energy functional, Iwamatsu $^{11}$ also obtained the same expression for the density profile. However, in his results $\zeta_{b}$ can be larger then 1 , which is unphysical. In our case, the fact that $\zeta_{b}$ is always less than 1 is consistent with the precondition required for the summation in Eq. (39).

Finally, to determine the unknown parameters $c_{1}$ and $c_{2}$ in (42) we used the condition of continuity of density profile and its derivative at the patching point $r_{0}$, which gives

$$
\begin{aligned}
& c_{1}=\left(\rho_{2}-\rho_{1}\right) \frac{1-\tanh \left(\zeta_{2} \lambda r_{0}\right) /\left(\zeta_{2} \lambda r_{0}\right)}{1+\left(\zeta_{1} / \zeta_{2}\right) \tanh \left(\zeta_{2} \lambda r_{0}\right)} \exp \left(\zeta_{1} \lambda r_{0}\right) r_{0}, \\
& c_{2}=\left(\rho_{2}-\rho_{1}\right) \frac{1+\zeta_{1} \lambda r_{0}}{1+\left(\zeta_{1} / \zeta_{2}\right) \tanh \left(\zeta_{2} \lambda r_{0}\right)} \frac{1}{\zeta_{2} \lambda \cosh \left(\zeta_{2} \lambda r_{0}\right)} .
\end{aligned}
$$

The only unknown quantity in (43) is the patching point $r_{0}$. Note that in the situation of the planar liquid-vapor interface the patching point $z_{0}$ can be simply determined via choosing a proper dividing surface (e.g., the equimolar dividing surface). Moreover, in the planar case, the density profile $\rho(z)$ does not depend on any external conditions and can be arbitrarily shifted along the $z$ axis. For small spherical droplets, however, the position of the patching point $r_{0}$ depends on droplet size. The latter is also a function of the supersaturation of vapor or the chemical potential. In the next section, our effort will be centered on finding the dependence of $r_{0}$ on the chemical potential, as well as the dependence of the surface tension of the spherical droplet on the radius.

\section{SURFACE TENSION OF A SPHERICAL DROPLET}

First, from (42) and (43) we can easily obtain the density at the patching point $r_{0}$,

$$
\rho\left(r_{0}\right)=\rho_{1}+\left(\rho_{2}-\rho_{1}\right) \frac{1-\tanh \left(\zeta_{2} \lambda r_{0}\right) /\left(\zeta_{2} \lambda r_{0}\right)}{1+\left(\zeta_{1} / \zeta_{2}\right) \tanh \left(\zeta_{2} \lambda r_{0}\right)},
$$

and density at the center of the droplet $r=0$,

$\rho(0)=\rho_{2}-\left(\rho_{2}-\rho_{1}\right) \frac{1+\zeta_{1} \lambda r_{0}}{1+\left(\zeta_{1} / \zeta_{2}\right) \tanh \left(\zeta_{2} \lambda r_{0}\right)} \frac{1}{\cosh \left(\zeta_{2} \lambda r_{0}\right)}$.

Equation (45) shows that $\rho(0)$ is always smaller than the density of uniform liquid $\rho_{2}$, and only in the case of an infinitely large droplet $\left(r_{0} \rightarrow \infty\right) \rho(0)=\rho_{2}$. This behavior was previously called the self-overlapping of the surface layer, ${ }^{7}$ which means that if the droplet is small the density profile inside the droplet is mainly that of interface and the profile never assumes the value of bulk liquid density. Similar behavior also takes place when a thin liquid film ${ }^{14}$ is condensed on the surface of a macroscopic particle, i.e., in the process of heterogeneous nucleation. However, for a large droplet the density at the center of the droplet can be larger than the density of the uniform liquid at coexistence 
$\rho_{(0) 2}$ due to compressibility. On the other hand, when $r_{0}$ $\rightarrow 0, \rho(0) \rightarrow \rho_{1}$ (the density of the supersaturated vapor) and $r_{0}$ becomes zero at gas spinodal. Beyond the gas spinodal, only uniform liquid can exist (liquid droplet cannot).

Next, let $\Delta \mu$ represent the chemical potential difference from saturation. Thus, $\Delta \mu=0$ for planar vapor-liquid interface and $\Delta \mu=\Delta \mu_{s}$ at the gas spinodal where $r_{0}=0$. Assuming $\rho\left(r_{0}\right)-\rho_{1}$ is a linear function of the chemical potential, an assumption similar to the double parabola approximation for the free energy used by Iwamatsu, ${ }^{11}$ the derivative $\partial \mu\left(\rho_{b}\right) / \partial \rho$ can then be considered as a constant $\gamma_{b}$, i.e., $\partial \mu\left(\rho_{b}\right) / \partial \rho=\partial \mu\left(\rho_{(0) b}\right) / \partial \rho \equiv \gamma_{b}$. The latter equality holds at all liquid and vapor regions, except near the spinodal point where $\partial \mu\left(\rho_{b}\right) / \partial \rho$ changes considerably. If we still enforce the equality in this region, we would overestimate the chemical potential at the spinodal but would have a complete model (including the spinodal point) for the derivative. From the above equality we have $\rho_{b}=\rho_{(0) b}+\Delta \mu / \gamma_{b}$, where $\gamma_{b}$ can be calculated from the case of planar-liquid interface.
With above assumptions we also have $\rho\left(r_{0}\right)-\rho_{1}$ $=a \Delta \mu+b$ where two constants $a$ and $b$ can be determined from two special conditions: (1) at $\Delta \mu=0$ we have the patching model for the density profile of planar liquid-vapor interface, i.e., $\rho\left(r_{0}\right)-\rho_{1}=\left(\rho_{(0) 2}-\rho_{(0) 1}\right) \zeta_{2} /\left(\zeta_{1}+\zeta_{2}\right)$, and (2) at $\Delta \mu=\Delta \mu_{s}, \rho\left(r_{0}\right)-\rho_{1}=0$. Using these two conditions, Eq. (44) can be written as

$$
\begin{aligned}
& \left(\rho_{(0) 2}-\rho_{(0) 1}\right) \frac{\zeta_{2}}{\zeta_{1}-\zeta_{2}}\left(1-\frac{\Delta \mu}{\Delta \mu_{s}}\right) \\
& \quad=\frac{1-\tanh \left(\zeta_{2} \lambda r_{0}\right) /\left(\zeta_{2} \lambda r_{0}\right)}{1+\left(\zeta_{1} / \zeta_{2}\right) \tanh \left(\zeta_{2} \lambda r_{0}\right)}\left[\rho_{(0) 2}-\rho_{(0) 1} \Delta \mu\left(\gamma_{2}^{-1}\right.\right. \\
& \left.\left.\quad-\gamma_{1}^{-1}\right)\right],
\end{aligned}
$$

from which we obtain the dependence of chemical potential of the droplet on the size of droplet $r_{0}$

$$
\Delta \mu=\left(\rho_{(0) 2}-\rho_{(0) 1}\right) \frac{\frac{\zeta_{2}}{\zeta_{1}+\zeta_{2}}-\frac{1-\tanh \left(\zeta_{2} \lambda r_{0}\right) /\left(\zeta_{2} \lambda r_{0}\right)}{1+\left(\zeta_{1} / \zeta_{2}\right) \tanh \left(\zeta_{2} \lambda r_{0}\right)}}{\frac{\rho_{(0) 2}-\rho_{(0) 1}}{\Delta \mu_{s}} \frac{\zeta_{2}}{\zeta_{1}+\zeta_{2}}+\left(\gamma_{2}^{-1}-\gamma_{1}^{-1}\right) \frac{1-\tanh \left(\zeta_{2} \lambda r_{0}\right) /\left(\zeta_{2} \lambda r_{0}\right)}{1+\left(\zeta_{1} / \zeta_{2}\right) \tanh \left(\zeta_{2} \lambda r_{0}\right.}}
$$

In Eq. (47) there is only one unknown quantity, $\Delta \mu_{s}$, the chemical potential difference at the spinodal. To obtain this quantity we invoke the asymptotic behavior of the chemical potential for large droplets, which can be described under the capillarity approximation as in classical nucleation theory

$$
\Delta \mu \sim \frac{2 \sigma_{\infty}}{r_{0}\left(\rho_{(0) 2}-\rho_{(0) 1}\right)} .
$$

Also from Eq. (47) we have, for large droplets,

$$
\Delta \mu \sim \frac{\left(\rho_{(0) 2}-\rho_{(0) 1}\right)}{\zeta_{2} \lambda r_{0}}\left(\frac{\rho_{(0) 2}-\rho_{(0) 1}}{\Delta \mu_{s}}+\gamma_{2}^{-1}-\gamma_{1}^{-1}\right)^{-1} .
$$

Combining Eqs. (48) and (49) gives

$$
\Delta \mu_{s}=\left(\rho_{(0) 2}-\rho_{(0) 1}\right)\left(\frac{\left(\rho_{(0) 2}-\rho_{(0) 1}\right)^{2}}{2 \sigma_{\infty} \zeta_{2} \lambda}-\gamma_{2}^{-1}+\gamma_{1}^{-1}\right)^{-1},
$$

and substituting Eq. (50) back into Eq. (47) yields

$$
\Delta \mu=\left(\rho_{(0) 2}-\rho_{(0) 1}\right) \frac{\frac{\zeta_{2}}{\zeta_{1}+\zeta_{2}}-\frac{1-\tanh \left(\zeta_{2} \lambda r_{0}\right) /\left(\zeta_{2} \lambda r_{0}\right)}{1+\left(\zeta_{1} / \zeta_{2}\right) \tanh \left(\zeta_{2} \lambda r_{0}\right)}}{\frac{\left(\rho_{(0) 2}-\rho_{(0) 1}\right)^{2}}{2 \sigma_{\infty}\left(\zeta_{2}+\zeta_{1}\right) \lambda}-\left(\gamma_{2}^{-1}-\gamma_{1}^{-1}\right)\left(\frac{\zeta_{2}}{\zeta_{1}+\zeta_{2}}-\frac{1-\tanh \left(\zeta_{2} \lambda r_{0}\right) /\left(\zeta_{2} \lambda r_{0}\right)}{1+\left(\zeta_{1} / \zeta_{2}\right) \tanh \left(\zeta_{2} \lambda r_{0}\right)}\right)} .
$$

Equation (51) is a central result in this section. It describes the dependence of chemical potential on the radius $r_{0}$ of the droplet. Note that $r_{0}$ cannot be identified as the radius of a realistic dividing surface as $R_{e}$ for the equimolar dividing surface or $R_{s}$ for the surface of tension. To find out the connection between $r_{0}$ and $R_{e}$ or $R_{s}$, we used the definition of the radius of equimolar dividing surface

$$
R_{e}^{3}=\frac{1}{\left(\rho_{1}-\rho_{2}\right)} \int_{0}^{\infty} r^{3} \frac{d \rho(r)}{d r} d r .
$$

Substituting the density profile of Eq. (42) into (52) yields

$$
\begin{aligned}
R_{e}^{3}= & r_{0}^{3}+3 r_{0}\left(1+\zeta_{1} \lambda r_{0}\right)\left[\left(\zeta_{1} \lambda\right)^{-2}-\left(\zeta_{2} \lambda\right)^{-2}\right] \\
& \times \frac{1-\tanh \left(\zeta_{2} \lambda r_{0}\right) /\left(\zeta_{2} \lambda r_{0}\right)}{1+\left(\zeta_{1} / \zeta_{2}\right) \tanh \left(\zeta_{2} \lambda r_{0}\right)} .
\end{aligned}
$$

Equations (51) and (53) allow us to obtain the dependence of the chemical potential on $R_{e}$. This dependence, where $r_{0}$ plays the role of auxiliary parameter, is plotted in Fig. 4. In Fig. 4 we also plot the dependence of chemical potential on 


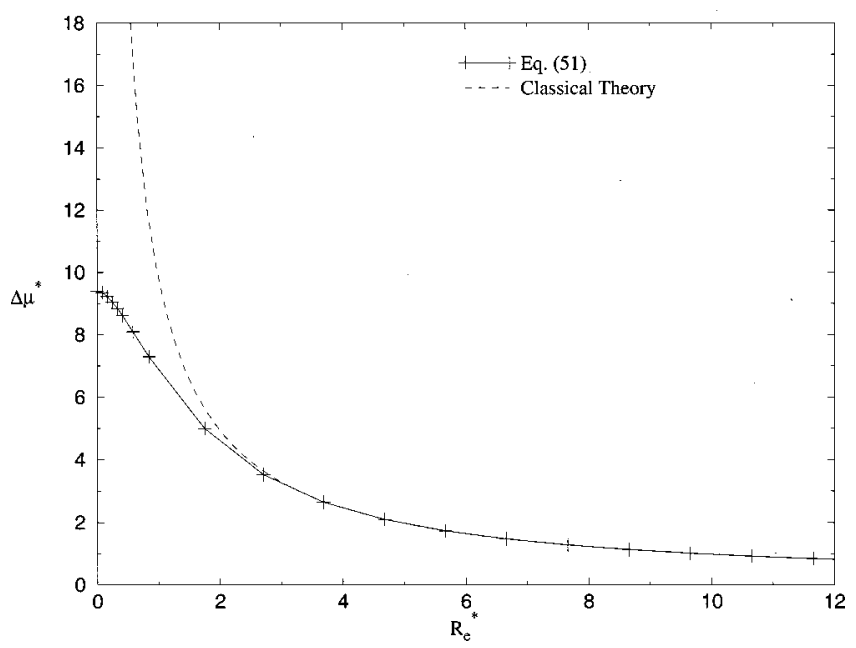

FIG. 4. The scaled chemical potential of the liquid droplet $\Delta \mu^{*}$ $=\Delta \mu / k_{B} T$ versus the reduced equimolar radius of the droplet $R_{e}^{*}=R_{e} / d$ at $T / T_{c}=0.4$.

the radius of the droplet derived from the classical capillarity approximation; that is, $\Delta \mu=2 \sigma_{\infty} /\left(\rho_{(0) 2}-\rho_{(0) 1}\right) R_{e}$. As shown in Fig. 4, marked differences can be found in the behavior of chemical potential from the classical nucleation theory and that from Eq. (51), and the differences become even larger for small droplets. In fact, as $R_{e} \rightarrow 0$ one curve approaches a limiting value $\Delta \mu_{s}$ while the other goes to infinity. This behavior of chemical potential was found previously in the framework of DFT theory. ${ }^{7}$ Similar behavior also occurs in the case of thin liquid film condensing on the surface of a macroscopic particle. There, the behavior of the chemical potential was explained by the presence of disjoining pressure in the thin liquid film. ${ }^{14}$

Last, we propose an analytical approach to estimate the surface tension of a droplet. To calculate the surface tension from DFT the usual approach is from Eq. (32). To do so, however, one needs to calculate the work of critical droplet formation, $\Omega+p_{1} V$, using a numerical method. ${ }^{3,4,7}$ Here, our analytical approach starts from the Laplace equation (20)

$$
\sigma_{s}\left(R_{s}\right)=\left(p_{2}-p_{1}\right) R_{s} / 2 .
$$

Given the approximations made above, it is not unreasonable to write the pressure difference $p_{2}-p_{1}$ in the form

$$
p_{2}-p_{1}=\Delta \mu\left(\rho_{(0) 2}-\rho_{(0) 1}\right)+\frac{(\Delta \mu)^{2}}{2}\left(\gamma_{2}^{-1}-\gamma_{1}^{-1}\right) \text {. }
$$

Given $p_{2}-p_{1}$ from Eq. (55), only one unknown quantity remains in Eq. (54), i.e., the radius of the surface of tension $R_{s}$. For the latter, a thermodynamic formula is given by

$$
R_{s}^{3}=\frac{3\left(\Omega+p_{1} V\right)}{2 \pi\left(p_{2}-p_{1}\right)}
$$

However, we cannot directly use Eq. (56) since we do not have $\Omega+p_{1} V$. To find $R_{s}$, we consider two limits: the large and small droplet limits.

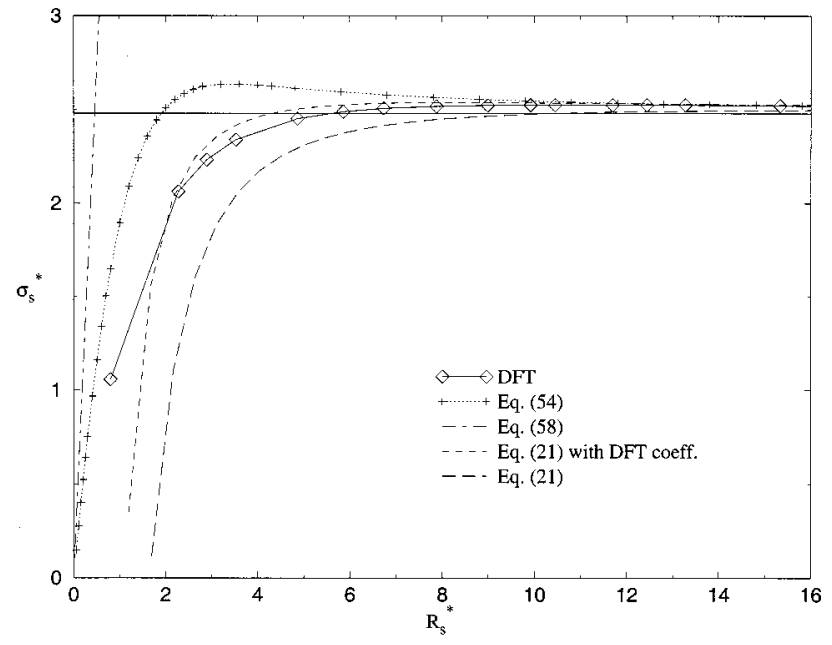

FIG. 5. The scaled surface tension at the surface of tension $\sigma_{s}^{*}=(\pi / 6)$ $\times\left(d^{2} / k_{B} T\right) \sigma_{s}$ versus the reduced radius of tension $R_{s}^{*}=R_{s} / d$ at $T / T_{c}$ $=0.4$. Horizontal line shows the surface tension of planar liquid-vapor interface.

In the limit of infinitely large droplet, the Tolman's formula $R_{e}-R_{s}=\delta_{\infty}$ is valid. Also in this limit, the density profile (13) gives $R_{e}=r_{0}-d\left(\zeta_{1}-\zeta_{2}\right) / \zeta_{1} \zeta_{2}$. Combining these two equations results in

$$
R_{s}=r_{0}-d \frac{\zeta_{1}-\zeta_{2}}{\zeta_{1} \zeta_{2}}-\delta_{\infty}
$$

Combining four equations, (51), (54), (55), and (57), gives a parametric dependence (with the parameter $r_{0}$ ) of surface tension $\sigma_{s}$ on $R_{s}$. This dependence is plotted in Fig. 5. Note that this dependence works only for sufficiently large droplets, for which the Tolman length can be considered as a constant $\left(\delta_{\infty}\right)$. The curve from exact DFT calculation [using formulas (32) and (56)] is also shown in Fig. 5. One can see that these two curves are close to each other in the region of large droplets. Despite many simplifications and assumptions, the two curves show qualitative agreement even in the region of small droplets.

In the second limit - the small droplet limit $\left(R_{s} \rightarrow 0\right)$, we do not have DFT results because the density profile of the critical nucleus becomes very unstable near the gas spinodal. ${ }^{3,4,7}$ Indeed, DFT cannot be applied for $R_{s} \sim 0$ where droplets consist of only a few particles. On the other hand, from Eq. (54) the behavior of the surface tension near $R_{s}$ $=0$ can be analyzed. If $R_{s} \rightarrow 0$, the chemical potential $\Delta \mu$ $\rightarrow \Delta \mu_{s}$ and the pressure difference $p_{2}-p_{1}$ approaches a finite limiting value $\Delta p_{s}=\Delta \mu_{s}\left(\rho_{(0) 2}-\rho_{(0) 1}\right)$ $+\left(\Delta \mu_{s}\right)^{2} / 2\left(\gamma_{2}^{-1}-\gamma_{1}^{-1}\right)$ according to Eq. (55), where $\Delta \mu_{s}$ is given by Eq. (50). Thus, the behavior of the surface tension near $R_{s}=0$ can be described by a linear function of $R_{s}$

$$
\sigma_{s}\left(R_{s}\right) \sim \Delta p_{s} R_{s} / 2 .
$$

Similar linear dependence of surface tension on the radius of the droplet for small droplets was first mentioned by Baidakov ${ }^{9}$ and Rusanov. ${ }^{15}$ As shown in Fig. 5, such a linear dependence is valid only near $R_{s}=0$.

In Fig. 5 two other curves are also shown, both from Eq. (21), but one is based on exact DFT and the other based on 
patching model values of coefficients $\delta_{\infty}$ and $\kappa$ (in both cases, exact DFT value of $\sigma_{\infty}$ is used). One can see that these two curves, especially the one based on exact DFT coefficients, describe the surface tension of large droplets quite well. In fact, the formula of surface tension [Eq. (21)] has been used more often than the one given by Eq. (54), but this formula works only when the expansion about small curvature is valid, whereas Eq. (54) provides a qualitative description of the surface tension for droplet with any size. From a quantitative point of view, Eq. (54) has a problem in that the maximum value it gives is in the region of too-small size of droplets, while Eq. (21) gives the maximum in the region of too-large droplets. We conclude that only Eq. (21) plus the numerical value of coefficients from DFT is capable of giving an accurate value of this maximum, compared to the one from numerical DFT calculation.

\section{CONCLUSION}

A patching model for the density profile of the spherical droplet based on analytical expressions for the asymptote of the profile is developed. Two possible models have been described. One is based on the functional expansion of the density profile about the small curvature and is therefore especially useful for very large droplets. Another patching model, although much more complex, can be used for spherical droplets of various size. The first model allows us to obtain analytical expressions for the surface tension of planar liquid-vapor interface, the Tolman length, and the effective rigidity constant. In spite of numerous assumptions and simplifications, the resulting analytical expressions still provide a good description, compared to the numerical DFT results, of temperature dependence of surface quantities over a wide range of temperatures.

As found in many previous work, ${ }^{1,5,6,10,16,17}$ we also find that the Tolman length is a small negative quantity with its absolute value weakly increasing with temperature. A second formula for the Tolman length is presented in Eq. (27), which is a special case of a more general expression for the Tolman length as mentioned in Paper I. The effective rigidity constant is also found to be a negative quantity with absolute value increasing with temperature.

The negative signs of the Tolman length and effective rigidity constant give rise to a nonmonotonic behavior of the surface tension. This nonmonotonic behavior was also pointed out in several papers based on DFT approach. ${ }^{5-7}$
Expressions for the Tolman length and effective rigidity constant allows us to develop a description of the dependence of surface tension on the radius of the droplet in the case of large droplets. This dependence is in good agreement with the exact DFT results for large droplets.

The second patching model for the density profile of the spherical droplet allows us to obtain the difference between the density in the center of the droplet and its value in the bulk liquid. It turns out that this difference results in significant changes in the behavior of chemical potential of small droplets, compared to that from the thermodynamic theory of nucleation based on the capillarity approximation. Finally, the dependence of the surface tension of the spherical droplet on the radius is also obtained. This dependence is quantitatively in good agreement with that from numerical DFT calculations for large droplets, but qualitatively in good agreement for small droplets. This dependence also demonstrates a nonmonotonic behavior of the surface tension.

\section{ACKNOWLEDGMENTS}

We are grateful to Professor Alexander Shchekin for useful discussions. X.C.Z. thanks the National Science Foundation for its support.

${ }^{1}$ T. Bykov and X. C. Zeng, J. Chem. Phys. 111, 3705 (1999).

${ }^{2}$ R. Becker and W. Döring, Ann. Phys. (Leipzig) 24, 719 (1935).

${ }^{3}$ D. W. Oxtoby and R. Evans, J. Chem. Phys. 89, 7521 (1988).

${ }^{4}$ X. C. Zeng and D. W. Oxtoby, J. Chem. Phys. 94, 4472 (1991).

${ }^{5}$ V. Talanquer and D. W. Oxtoby, J. Phys. Chem. 99, 2865 (1995).

${ }^{6}$ K. Koga, X. C. Zeng, and A. K. Shchekin, J. Chem. Phys. 109, 4063 (1998)

${ }^{7}$ T. V. Bykov and A. K. Shchekin, Colloid J. USSR 61, 144 (1999).

${ }^{8}$ E. M. Blokhuis and D. Bedeaux, Physica A 184, 42 (1992).

${ }^{9}$ V. G. Baidakov and G. Sh. Boltachev, Russ. J. Phys. Chem. 69, 515 (1995); V. G. Baidakov and G. Sh. Boltachev, Phys. Rev. E 59, 469 (1999).

${ }^{10}$ T. V. Bykov and A. K. Shchekin, Inorg. Mater. (Transl. of Neorg. Mater.) 35, 641 (1999).

${ }^{11}$ M. Iwamatsu, J. Phys.: Condens. Matter 5, 7537 (1993); 6, L173 (1994).

${ }^{12} \mathrm{R}$. Evans, in Fundamentals of Inhomogeneous Fluids, edited by D. Henderson (Wiley, New York, 1992).

${ }^{13}$ E. M. Blokhuis and D. Bedeaux, Mol. Phys. 80, 705 (1993).

${ }^{14}$ F. M. Kuni, A. K. Shchekin, A. I. Rusanov, and B. Widom, Adv. Colloid Interface Sci. 65, 71 (1996).

${ }^{15}$ A. I. Rusanov, Fasovye Ravnoveesiya i Poverkhnostnie Yavleniya (Khimia, Leningrad, 1967).

${ }^{16}$ A. E. van Giessen, E. M. Blokhuis, and D. J. Bukman, J. Chem. Phys. 108, 1148 (1998).

${ }^{17}$ K. Koga and X. C. Zeng, J. Chem. Phys. 110, 3466 (1999). 